\title{
Multicore Fiber Opportunities for Centralized Radio Access Networks
}

\author{
Ivana Gasulla and Joaquín Pérez \\ ITEAM Research Institute, Universitat Politecnica de Valencia, 46022 Valencia, Spain \\ Tel: (34)963877007,e-mail: ivgames@iteam.upv.es
}

\begin{abstract}
Radio-over-fiber architectures conceived for the next generation of wireless access systems are based on Centralized Radio Access Networks (C-RAN), where the baseband processing resources corresponding to different remote base stations (BS) are hosted in a shared central office (CO). We propose the exploitation of the unique features brought by the inherent parallelism of multicore fibers as a compact solution to implement the required CO-BS connectivity in C-RANs.
\end{abstract}

Keywords: microwave photonics, radio-over-fiber, radio access networks, multicore fibers, space-division multiplexing.

\section{INTRODUCTION}

Centralized or Cloud Radio Access Networks (C-RANs) have been recently proposed as the forthcoming evolution of broadband converged fiber-wireless access networks, [1]-[4]. Compared to traditional RAN architectures, such as the one shown in Fig. 1, composed of many stand-alone macro base transceiver stations (BTS) where all the optical and electrical processing equipment is located at each tower base; C-RAN configurations, as shown in Fig. 2, benefit from hosting the baseband processing resources corresponding to different remote BTSs in a shared Central Office (CO). This centralized configuration implies the introduction of a fronthaul segment for the remote feeding of different BTS antennas, which may in addition feature multiple radiating elements per section improving the capabilities of coordination between them.

The fronthaul segment of this architecture, implemented with digital radio-over-fiber (DRoF) links, can benefit from the parallelism offered by optical Space-Division Multiplexing (SDM) technologies, [6],[7]. We propose in this paper the novel use of multicore fibers (MCFs) as a compact solution to implement the distributed DRoF connectivity with the required degree of parallelism between the $\mathrm{CO}$ and the set of remote BTSs, which, in turn, may be composed of sectorized antennas featuring spatial wireless MIMO. MCFs are behind current world capacity-transmission records in SDM-based transport networks, but their use in the context of RANs has not yet been considered to the best of our knowledge. In particular, we address the use of homogeneous multicore fibers for DRoF distribution and consider as well the use of heterogeneous MCFs for additional signal processing functionalities, such as optical beamforming.

\section{EVOLUTION TOWARDS CENTRALIZED RADIO ACCESS NETWORKS}

Traditional RAN architectures, mostly deployed in macro-cell $1 \mathrm{G}$ and $2 \mathrm{G}$ cellular networks, are composed of many stand-alone BTSs that are connected to the CO through an optical fiber backhaul segment, usually composed of a set of point-to-point single-core single-mode fiber (SMF) links. As shown in Fig. 1, the equipment that must be placed in each one of these all-in-one BTSs includes three different parts [1]-[4]. The first is the radiating system, usually a three-sector antenna site. The second is a separate cell site cabinet (CSC) that hosts the main equipment of the BTS, including the remote radio units (RRUs), the baseband unit (BBU), a small DRoF segment connecting the RRUs to the BBU and the system module. Finally, the third is the cell site gateway (CSG) that interfaces the CSC to the backhaul network. In the BTS, the downstream signal received from the $\mathrm{CO}$ through the backhaul network is in baseband format. It is processed by the BBU, up-converted to radio, translated to the optical domain, and sent to the three different RRUs by means of three different DRoF links. At the RRUs, the signal is down-converted again to the radiofrequency (RF) domain and sent through pairs of RF coaxial cables to the antennas on the BTS tower where it is radiated. The same operations in reversed order are performed for the received upstream radio signal.

This traditional RAN architecture has several important drawbacks. In the first place, the addition of more BTSs to improve capacity, results in a severe interference among BTSs since they share the same frequency resource and must be placed closer to each other. Secondly, each BTS is actually costly to build and operate, as it needs a considerable number of autonomous equipment, including its own cooling, backhaul transportation, backup battery, monitoring system, etc. Finally, because mobile users are moving from one place to another, the traffic managed by each BTS experiences considerable time fluctuations and, as a result, the average utilization rate of individual BTSs is pretty low.

(C) 2014 IEEE. Personal use of this material is permitted. Permission from IEEE must be obtained for all other uses, in any current or future media, including reprinting/republishing this material for advertising or promotional purposes, creating new collective works, for resale.

Published at: 2014 16th International Conference on Transparent Optical Networks (ICTON) by IEEE,

Published version available at DOI: https://doi.org/10.1109/ICTON.2014.6876553 


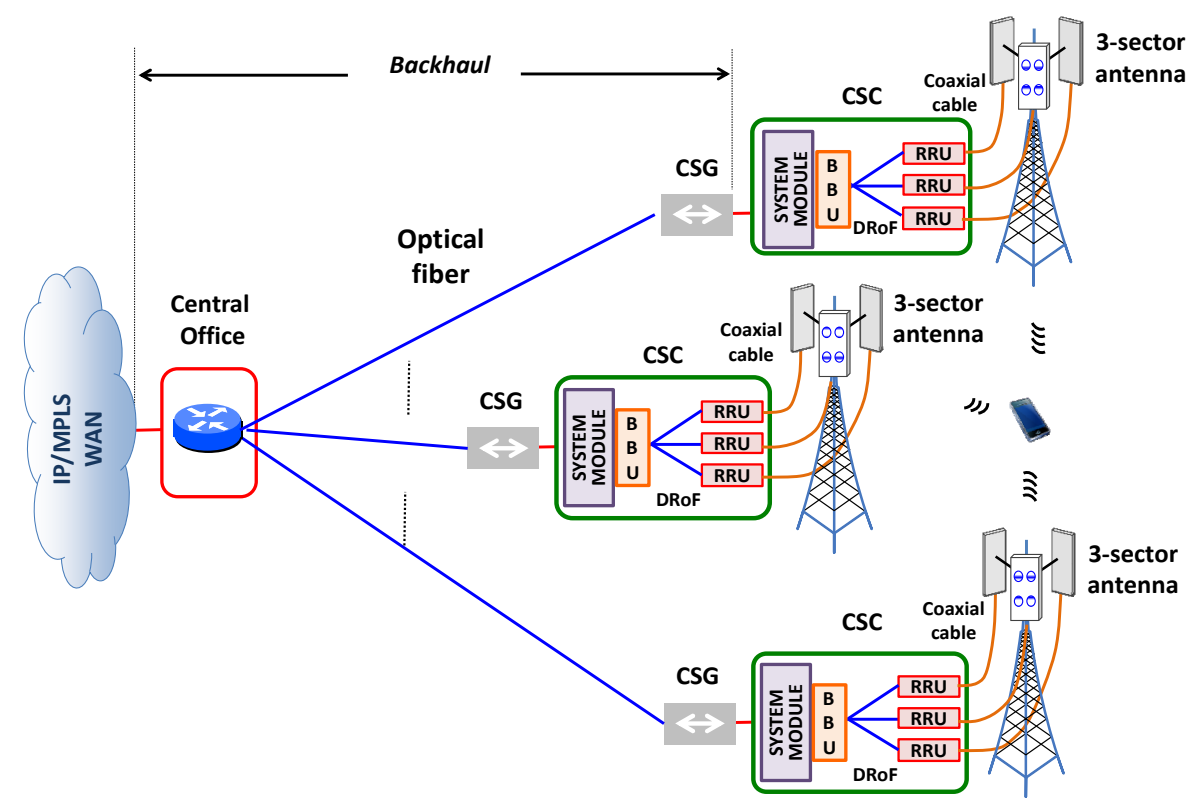

Figure 1. Architecture of a traditional RAN showing the backhaul segment.

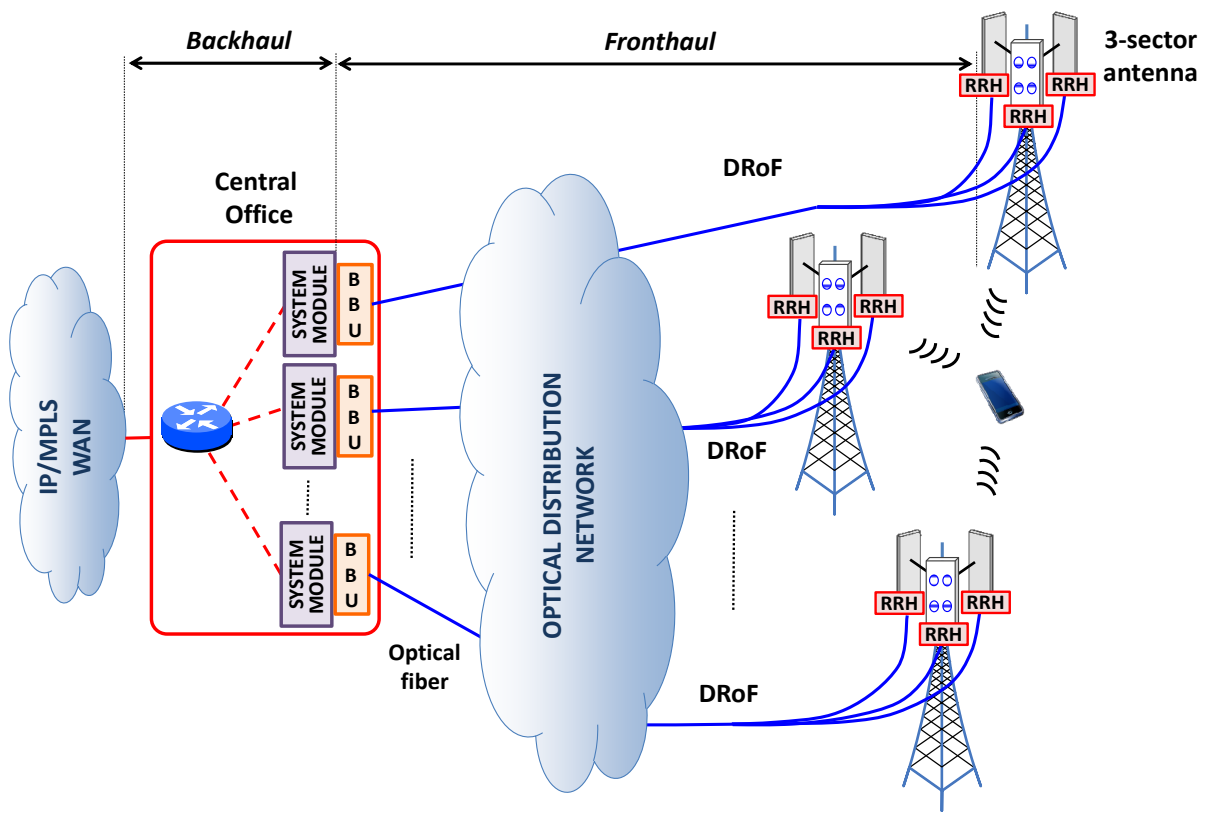

Figure 2. Architecture of a Centralized RAN showing the fronthaul and backhaul segments.

Much of the above limitations can be solved if many of the baseband processing resources are shared among various BTSs. This has led the evolution towards the so-called C-RANs, where the BBU equipment is hosted in the CO rather than at the remote BTS locations, [1],[4]. These resource hostelling can be implemented either by BBU stacking, as it is shown in Fig. 2, or by BBU pooling where a single BBU manages several antennas.

The newly introduced fronthaul segment connects the RRUs, now called remote radiating heads (RRHs), feeding the radiating elements with the centralized BBU/BBUs. A backhaul segment is also present in between the BBUs and the connection node to the transport Wide-Area Network (WAN). Several distinctive features characterize the C-RAN architecture compared to traditional cellular network architectures. In first place, as it builds upon a large-scale centralized deployment, it has the potential for allowing hundreds or even thousands of remote RRHs to be connected to a centralized BBU pool. This enables a higher coverage density from a single CO. The Common Public radio Interface (CPRI) is the digital interface standard for encapsulating the radio samples between the RRHs and the BBU. The radio signals are multiplexed in a low-latency timeslot-like technique, not packet-based, that limits the fronthaul link distances and defines a maximum latency of $0.4 \mathrm{~ms}$. This implies, in consequence, a fronthaul segment conceived to span maximum fiber link distances of up to $2 \mathrm{~km}$ for future $5 \mathrm{G}$ and $\mathrm{mm}$-Wave systems, $20 \mathrm{~km}$ for $4 \mathrm{G}$ (LTE/LTE-A) systems and 40-80 km for 3G (WCDMA/TDSCDMA) and 2G (GSM/CDMA) systems. A second advantage of C-RAN results from the ability of providing native support to collaborative multipoint (CoMP) radio technologies. Since any given BBU can communicate at 
very high speed $(10 \mathrm{~Gb} / \mathrm{s}$ and above) with very low latency with any other one hosted at the same pool, almost instantaneous coordination between different cells required for CoMP is guaranteed.

\section{MULTICORE FIBERS AS A DISTRIBUTION SOLUTION}

So far, the proposed designs for the C-RAN fronthaul distribution are composed by multiple parallel DRoF links implemented by single-core SMFs that individually feed each RRH unit placed at the BTS. Since sectorization techniques are implemented at the BTS antenna systems, ranging from the conventional 3-sector antennas to higher-order configurations (up to 12 sectors), multiple bidirectional fiber links must be deployed to each BTS tower. In addition, the use of multiple input multiple output (MIMO) radio transmission through the implementation of spatial diversity, beamforming and/or spatial multiplexing techniques, considerably increases the number of required DRoF connections, [5]. For instance, the LTE wireless standard supports up to 4 antennas for downlink transmission $(4 \times 4$ MIMO), whereas LTE-Advanced (LTE-A) enhanced MIMO transmission with a downlink extension to $8 \times 8$ and the introduction of uplink MIMO up to $4 \times 4$, [5]. The implementation of spatial MIMO designs, for both diversity and multiplexing techniques, increase in consequence the required degree of parallelism by a factor ranging from 4 to 8 , and can in consequence benefit from the inherent parallelism and compactness offered by MCFs. This implies a potential cost-saving deployment advantage not only over traditional SMF fronthaul schemes, where an independent SMF is required for each CO-RRH connection; but also over recently proposed active systems using Wavelength Division Multiplexing (WDM) techniques, where the number of required SMFs equals the number of antenna sections, [1].

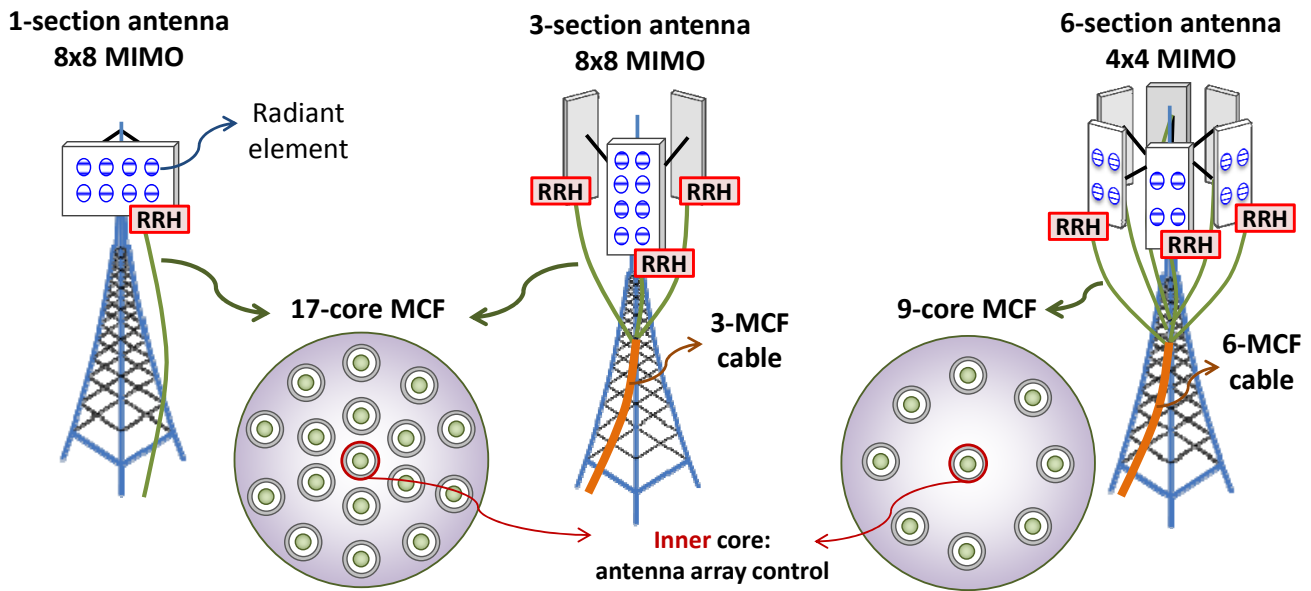

Figure 3. Digital RoF distribution schemes over MCF for sectorized antennas with spatial MIMO.

MCFs provide different propagation pathways implemented by a set of physically distinct single-mode cores that are confined inside a single cladding with an outer diameter ranging from 125 to $200 \mu \mathrm{m}$, [6]. Most of the research activity on SDM has employed homogeneous MCFs where all the cores are identical and thus share the same propagation characteristics. Cross coupling between the cores and the resulting crosstalk can be reduced by keeping the cores well-spaced and further mitigated by introducing trench-assisted configurations around each core. For most radio signal modulation formats, a crosstalk level below $-25 \mathrm{~dB}$ is enough to avoid significant transmission penalties. Previous work on SDM transport networks reported figures well below this value $(-50 \mathrm{~dB}$ for a bidirectional 450-km link) for homogeneous MCFs, [7]. Those results encourage the exploitation of MCFs not only for the transmission of radio, intermediate frequency (IF) and baseband data signals; but also for the remote optical carrier delivery required in upstream transmission as well as the remote delivery of RF and local oscillators. Figure 3 illustrates some potential scenarios for 1-, 3- and 6-section (implementing $4 \times 4$ and $8 \times 8$ MIMO schemes) BTS antennas where the bidirectional distribution of the 4 or 8 data signals is conducted by the outer cores of the homogeneous MCFs, while the inner core is devoted to a common control signal. As an example, Fig. 4 shows the fronthaul DRoF connection between a shared $\mathrm{CO}$ and a set of 8 RRH units conforming the $8 \times 8 \mathrm{MIMO}$ antenna sector of a representative remote BTS. Both the delivery of the downstream and upstream IF data signals and the remote feeding of the common local oscillator (LO) are performed over a single 17-core MCF.

As we have seen, resorting to homogeneous MCFs as a distribution solution in C-RANs provides in a single fiber the required distribution for BTS antennas implementing MIMO. In addition, the inherent parallelism of MCFs can be exploited for the generation and processing of microwave and millimeter-wave signals, as originally proposed in [8]. More precisely, heterogeneous MCFs, whose single cores present individual propagation characteristics, can be used to implement sampled discrete true time delay lines for RF signals. This Microwave Photonics (MWP) functionality is indeed the basis of the third type of MIMO that feature smart radiating systems, optical beamforming for phase-array antennas (PAAs), that allows to direct the signal energy 
in the direction of the user or BTS receiver. This implies that the proposed MCF-based fronthaul architecture could also benefit from MWP devices based on heterogeneous MCFs.

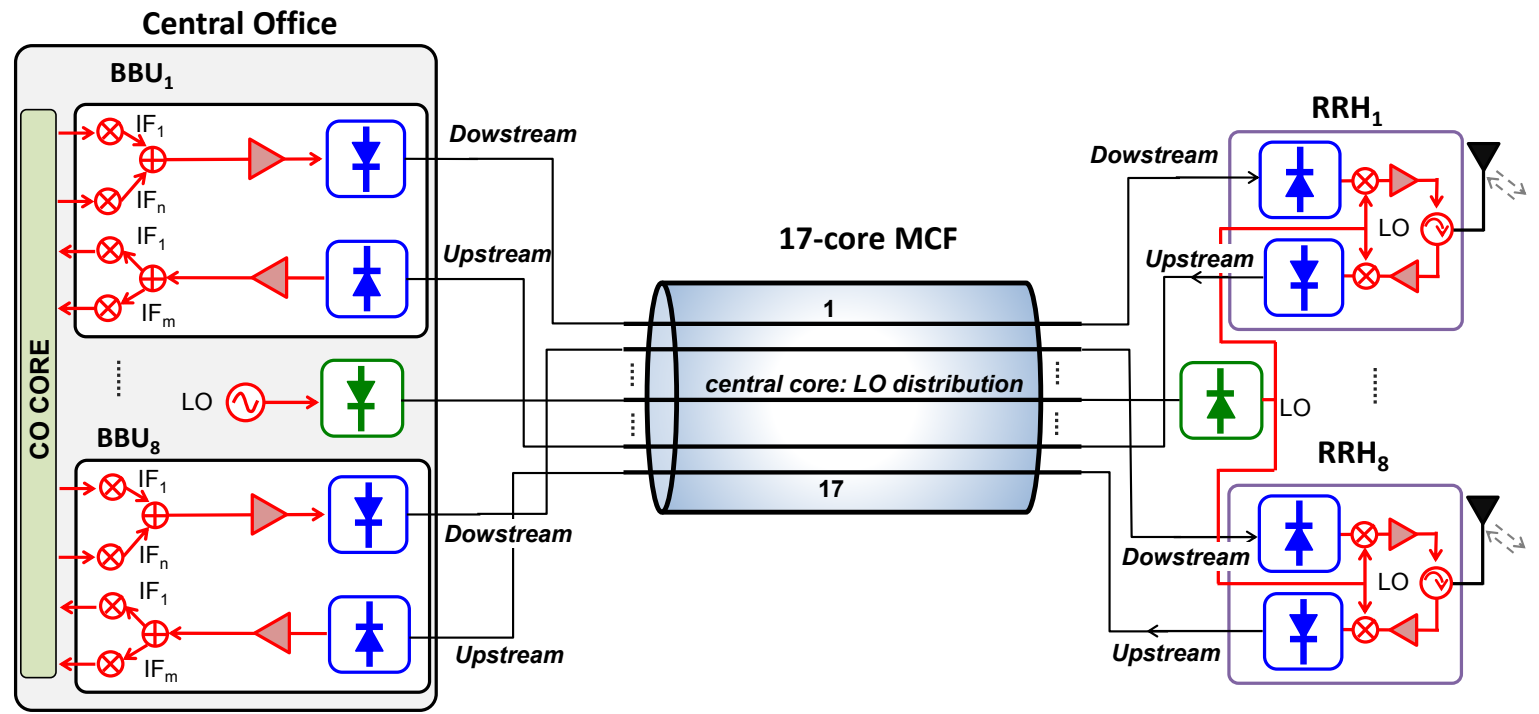

Figure 4. Remote IF data signals and LO feeding to RRH units using a 17-core MCF.

\section{CONCLUSIONS}

The forthcoming generation of Centralized Radio Access Networks can greatly benefit from the compactness and spatial parallelism offered by optical Space-Division multiplexing technologies. We envision that the branched and replicated distribution required in the DRoF fronthaul segment connecting the resource-centralized $\mathrm{CO}$ and the remote BTSs can directly be offered in a single homogeneous MCF, instead of compelled replication fiber architectures. This results in an essential cost-saving deployment advantage over previously proposed single-core single-mode fiber schemes that require the use of WDM techniques. Furthermore, additional MWP processing functionalities, in particular optical beamforming for PAAs, can also be implemented with heterogeneous MCFs, without the need to resort to bulky, expensive and power-consuming devices. The proposed converged MCF-wireless architectures will provide in consequence a high positive impact on the final cost of the next generation of C-RANs that will pave the way towards the deployment of LTE-A and the future $5 \mathrm{G}$ broadband access networks.

\section{ACKNOWLEDGEMENTS}

The authors wish to acknowledge the financial support given by the Research Excellency Award Program GVA PROMETEO II/2013/012 and by J. Perez's Spanish MINECO Juan de la Cierva Fellowship JCI-2012-14805.

\section{REFERENCES}

[1] P. Chanclou et al:: Optical fiber solution for mobile fronthaul to achieve cloud radio access network, in Proc. Future Network \& Mobile Summit Conf. 2013, Lisbon, Portugal, Jul. 2013.

[2] A. Pizzinat et al.: FTTx where x stays for antenna: Requirements on optical access/distribution network for new mobile backhaul architectures, in Proc. FTTx SUMMIT Europe, London, UK, Apr. 2012.

[3] A. Pizzinat et al.: Infrastructure convergence for fixed and mobile access networks, in Proc. OFC 2009, Workshop "Migration Scenarios toward Future Access Networks I", San Diego, USA, Mar. 2009.

[4] A. Saadani et al:: Digital radio over fiber for LTE-Advanced: Opportunities and challenges, in Proc. ONDM, Brest, France, April 2013, pp. 194-199.

[5] F. Boccardi et al.: Multiple-antenna techniques in LTE-advanced, IEEE Communications Magazine, vol. 50, pp. 114-121, 2012.

[6] D.J. Richardson, J.M. Fini, and L.E. Nelson: Space division multiplexing in optical fibers, Nature Photonics, vol. 7, pp. 354-362, 2013.

[7] A. Sano et al.: 409-Tb/s + 409-Tb/s crosstalk suppressed bidirectional MCF transmission over $450 \mathrm{~km}$ using propagation-direction interleaving, Opt. Express, vol. 21, pp. 16777-16783, 2013.

[8] I. Gasulla and J. Capmany: Microwave photonics applications of multicore fibers, Photonics J., vol. 4, pp. 877-888, 2012. 